\title{
Blp1 protein shows virulence-associated features and elicits protective immunity to Acinetobacter baumannii infection
}

\author{
Jūrate Skerniškytè ${ }^{1 *}$, Emilija Karazijaitè $^{1}$, Julien Deschamps², Renatas Krasauskas', Julija Armalytè
}

Romain Briandet ${ }^{2}$ and Edita Sužiedèliené ${ }^{1}$

\begin{abstract}
Background: Multidrug resistant Acinetobacter baumannii is one of the major infection agents causing nosocomial pneumonia. Therefore, new therapeutic approaches against this bacterium are needed. Surface-exposed proteins from bacterial pathogens are implicated in a variety of virulence-related traits and are considered as promising candidates for vaccine development.

Results: We show in this study that a large Blp1 protein from opportunistic pathogen A. baumannii is encoded in all examined clinical strains of globally spread international clonal lineages I (IC I) and II (IC II). The two blp1 gene variants exhibit lineage-specific distribution profile. By characterization of blp1 deletion mutants and their complementation with blp 1 alleles we show that blp 1 gene is required for $A$. baumannii biofilm formation and adhesion to epithelial cells in IC I strain but not in the IC II strain. Nevertheless both alleles are functional in restoring the deficient phenotypes of IC I strain. Moreover, the blp 1 gene is required for the establishing of $A$. baumannii virulence phenotype in nematode and murine infection models. Additionally, we demonstrate that C-terminal 711 amino acid fragment of Blp1 elicits an efficient protection to lethal $A$. baumannii infection in a murine model using active and passive immunization approaches. Antiserum obtained against Blp1-specific antigen provides opsonophagocytic killing of $A$. baumannii in vitro.
\end{abstract}

Conclusions: Lineage-specific variants of surface-exposed components of bacterial pathogens complicate the development of new therapeutic approaches. Though we demonstrated different impact of Blp1 variants on adherence of IC I and IC II strains, Blp1-specific antiserum neutralized A. baumannii strains of both clonal lineages. Together with the observed increased survival rate in vaccinated mice these results indicate that $A$. baumannii Blp1 protein could be considered as a new vaccine candidate.

Keywords: Acinetobacter baumannii, Blp1, Virulence, Biofilm, Vaccine

\section{Background}

Acinetobacter baumannii is a widely spread opportunistic pathogen causing pneumonia, sepsis and soft tissue infections [1]. A. baumannii is the most dangerous to critically ill patients and is responsible for hospital infection outbreaks, especially in the intensive care units $[2,3]$. Antibiotic therapy is complicated due to the global spread of

\footnotetext{
* Correspondence: jurate.skerniskyte@gf.vu.lt

${ }^{1}$ Institute of Biosciences, Life Sciences Center, Vilnius University, Saulètekio ave. 7, LT-10257 Vilnius, Lithuania

Full list of author information is available at the end of the article
}

multidrug-resistant (MDR) strains, non-susceptible to most antibiotic classes [4]. Therefore, A. baumannii has been considered as a top priority pathogen for which new drugs and therapeutic options are urgently needed [5].

Among strategies considered, the vaccine-based approaches show the greatest potential [6]. However, the main challenge to identify an efficient vaccine candidate against A. baumannii infections still remains [6]. The desirable antigens should be highly prevalent surfaceexposed bacterial proteins and possess high degree of conservation among clinical strains. However, the vast

(c) The Author(s). 2019 Open Access This article is distributed under the terms of the Creative Commons Attribution 4.0 International License (http://creativecommons.org/licenses/by/4.0/), which permits unrestricted use, distribution, and reproduction in any medium, provided you give appropriate credit to the original author(s) and the source, provide a link to the Creative Commons license, and indicate if changes were made. The Creative Commons Public Domain Dedication waiver (http://creativecommons.org/publicdomain/zero/1.0/) applies to the data made available in this article, unless otherwise stated. 
majority of clinical A. baumannii isolates possess a thick outer layer of polysaccharides (capsule), which efficiently protects pathogens from the host immunity by shielding cell surface antigens $[7,8]$. To be able to adhere to the host cells and form biofilms, capsulated pathogens use long extended molecules such as adhesins or pili-like structures, penetrating the thick capsule $[9,10]$. Exploring the characteristics of such surface-exposed proteins and their role in pathogenesis might reveal the new targets suitable for antimicrobial therapy against clinical $A$. baumannii MDR strains.

The A. baumannii Blp1 protein (also named as BapA, GenBank accession number: WP_126655828) has been recently identified to exhibit structural organization, similar to that of a giant A. baumannii protein called Bap (biofilm-associated protein) [11]. Blp1 exhibits a tripartite structure, which includes $\mathrm{C}$-terminal and $\mathrm{N}$-terminal domains, separated by a large portion of a repetitive region containing combinations of various motifs including bacterial Ig-like (Big) domains. The Big-like domains, present in both Blp1 and Bap proteins, are a characteristic feature of some bacterial fimbrial and non-fimbrial adhesins, which enhance the attachment to various materials, such as host cells or abiotic surfaces [9, 12-14]. Indeed, it has been demonstrated recently that the deletion of blp1 gene decreases A. baumannii biofilm formation and adhesion to the lung epithelial cells A549 [11]. Unlike the bap gene, which seems to be truncated in a number of $A$. baumannii genomes, the intact $b l p 1$ allele was common in $A$. baumannii genomes analyzed [11]. Therefore, the predicted bacterial surface-associated features of Blp1 protein and the prevalence of blp1 gene in bacterial genomes make Blp1 an attractive antigen candidate for vaccination-based strategies against MDR A. baumannii.

Two major A. baumannii pandemic clonal lineages, namely IC I and IC II, cause the highest percentage of hospital acquired $A$. baumannii infections worldwide [2, 3]. Genome analysis of the strains belonging to these two clonal lineages demonstrated the significant differences in genetic determinants of antimicrobial resistance [15-17]. Due to high carbapenem-resistance, the IC II lineage strains are characterized through the increased nosocomial spread in many countries during the recent years $[2,3]$. Since lineage-depended genetic differences were also observed in surface-related components [15-17], the substantial role of these specific genes variants in A. baumannii dissemination and pathogenesis could be assumed. The objective of this study was to investigate the incidence and distribution of blp1 gene variants among clinical A. baumannii strains, belonging to the most globally spread clonal lineages, to assess the role of blp1 gene in virulencerelated features and, most importantly, to evaluate the efficiency of Blp1 protein-based vaccine against $A$. baumannii in murine infection model.

\section{Results}

The distribution of blp1 alleles in clinical A. baumannii strains of international clonal lineages I and II

$A$. baumannii is characterized by a high degree of genomic variability [15-17] and this trait was recently demonstrated for blp1 gene [11]. Therefore, we were interested in the prevalence and variability of blp1 gene in the clinically relevant strains belonging to two predominant clones IC I and IC II. First, we have performed analysis of the genomes of strains with the assigned clonal lineage I or II, available in NCBI Genbank and have observed a clear association of two different blp1 allele variants with either IC I or IC II lineage strains (Fig. 1a). The number of bacterial Ig-like repeat units (Bigs) encoded in blp1 gene varied among the strains, typically 26 units found in gene variant in IC I strains and 24-25 units in its counterpart in IC II strains (Fig. 1b). Two types of repeats, named Big_3_2 and Big_6, were identified in both alleles. The Blp1 protein variants encoded in the genomes of IC I and IC II strains showed 71-74\% identity. Approximately 160 amino acids at the Blp1 C-

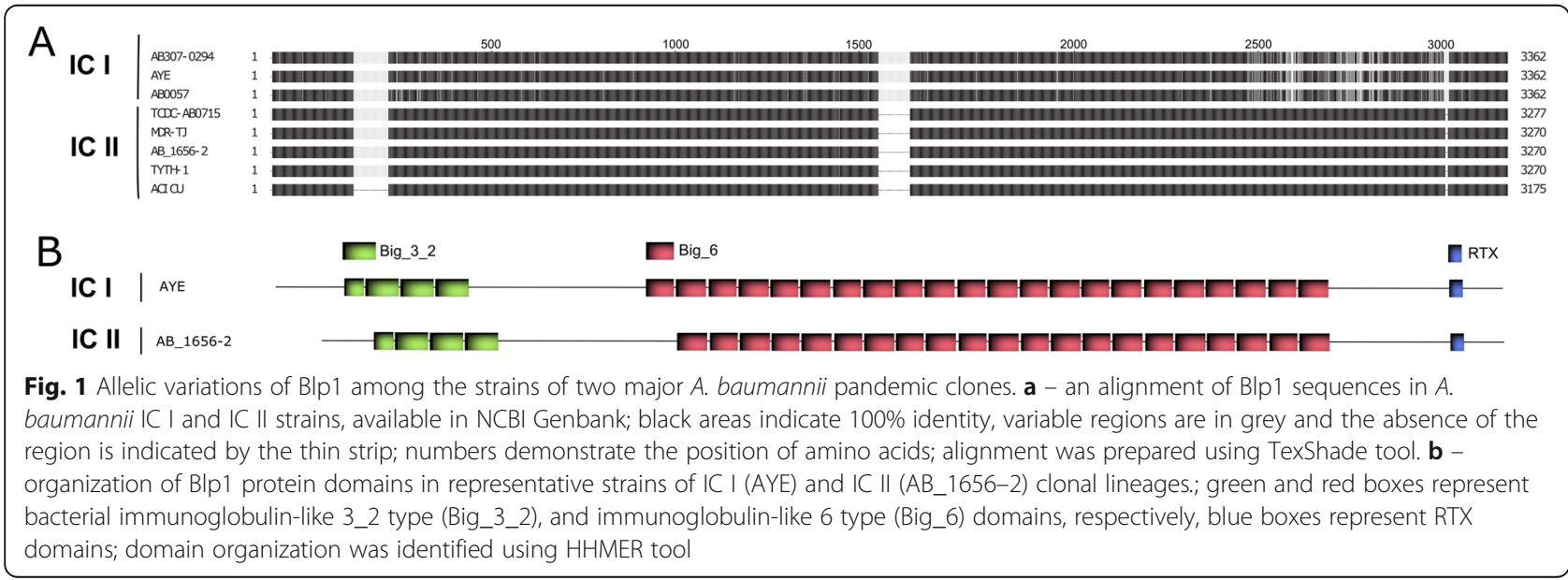


terminus harboring RTX-toxin domain and T1SS signal sequence were conserved in both protein variants. Whereas the region of approximately 540 amino acids between Big repeats and RTX-domain, contained IC-specific variations (Fig. 1a). The observed A. baumannii Blp1 C-terminal sequence variations, commonly found in bacterial surface exposed structures $[18,19]$, are in line with the proposed Blp1 surface association.

Next, to confirm the relationship of blp1 gene alleles with the IC lineages, we designed the primer pairs (Additional file 1: Table S1) specifically targeting each allele variant and screened for their prevalence in the collection of clinical A. baumannii isolates representing IC I $(n=72)$ and IC II $(n=50)$ clonal lineages. All tested IC I strains were found to possess $b l p 1_{\text {IC I }}$ allele, while all IC II strains contained $b l p 1_{\text {IC II }}$ allele, showing the distribution profile, strictly specific for clonal lineage, thereby confirming the trait observed from the in silico analysis (Fig. 1a).

\section{The loss of blp1 gene differently impacts the ability of $A$. baumannii IC I and IC II strains to form biofilm}

The observed blp1 polymorphism among IC I and IC II strains prompted us to investigate their functionality differences by assessing the virulence-related A. baumannii properties. For this purpose, two representative clinical A. baumannii isolates belonging to IC I and IC II clonal lineages, designated $\mathrm{Ab}_{\mathrm{IC}} \mathrm{I}$ and $\mathrm{Ab}_{\mathrm{IC}} \mathrm{II}$, respectively, were selected from the collection of previously characterized clinical isolates [20] and used for further analysis. Their characteristics are represented in Additional file 1: Table $\mathrm{S} 1$. The $A \mathrm{~b}_{\mathrm{IC} \text { I }}$ and $A \mathrm{~b}_{\mathrm{IC} \text { II }}$ strains were assigned to ST231 and ST208 sequence types, respectively, according to the Oxford MLST typing scheme. Both these sequence types were found to be globally disseminated [21, 22]. Markerless deletions of blp 1 allelic variants in $\mathrm{Ab}_{\mathrm{IC} \text { I }}$ and $\mathrm{Ab}_{\mathrm{IC}}$ II strains were generated as described in Materials and Methods. Notably, the blp1 deletions did not result in any alterations of the growth of mutant strains (data not shown). For the complementation experiments, plasmids $\mathrm{p} b l p 1_{\mathrm{IC}}$ and pblp $1_{\text {IC II }}$ carrying blp1 allelic variants were constructed and introduced into $\mathrm{Ab}_{\mathrm{IC}} \mathrm{I} \Delta b l p 1$ and $\mathrm{Ab}_{\mathrm{IC}}{ }_{\mathrm{II}} \Delta b l p 1$ strains as described in Materials and Methods. The loss of endogenous blp1 gene expression in the $\Delta b l p 1$ mutants and restoration of blp1 expression by complementation, has been verified by qPCR using primers BaprB/BapRaiR (Additional file 1: Table S1; Additional file 2: Figure S1).

Firstly, the biofilm formation of $\mathrm{Ab}_{\mathrm{IC}} \mathrm{I} \Delta b l p 1$ and $\mathrm{Ab}_{\mathrm{IC}}$ ${ }_{\text {II }} \Delta b l p 1$ mutants and their blp1-complemented strains was assessed. Bacteria were grown on the plastic surface and the formed structures were analyzed by confocal laser scanning microscopy (CLSM) using SYTO9 and propidium iodide (PI) staining as described in Materials and Methods. Interestingly, $2 \mathrm{~h}$ post-seeding, the initial attachment of bacteria to the plastic surface, based on the increase of PI-stained (red stain) versus total amount of bacteria (green stain) was affected in $\mathrm{Ab}_{\mathrm{IC}} \mathrm{I} b \mathrm{blpl} \mathrm{mu}$ tant, demonstrating up to $55 \%$ increase in PI-stained bacteria compared with the parental strain (Fig. 2a, upper panels). Contrary to the $\mathrm{Ab}_{\mathrm{IC}} \mathrm{I} \Delta b l p 1$ mutant, the $\mathrm{Ab}_{\mathrm{IC}}{ }_{\mathrm{II}} \Delta b l p 1$ strain showed no alterations in the initial attachment compared to the parent, evident through the lack of PI-stained bacteria and through the formation of more uniform biofilm structure similar to parental strain (Fig. 2a, lower panels). To verify that the observed phenotype of $\mathrm{Ab}_{\mathrm{IC}}{ }_{\mathrm{I}} \Delta b l p 1$ was indeed blp1-dependent, we introduced plasmids $\mathrm{p} b l p 1_{\mathrm{IC} \text { I }}$ and $\mathrm{p} b l p 1_{\mathrm{IC} \text { II }}$ carrying respective blp1 alleles into $\mathrm{Ab}_{\mathrm{IC}}{ }_{\mathrm{I}} \Delta b l p 1$ bacteria and assessed their attachment to the plastic surface. Notably, both blp1 alleles efficiently restored the phenotype of $\mathrm{Ab}_{\mathrm{IC}}{ }_{\mathrm{I}} \Delta b l p 1$ mutant manifested by the reduction in PIstained cells compared to the $\mathrm{Ab}_{\mathrm{IC}} \mathrm{I} \Delta b l p 1$ mutant carrying control plasmid (Fig. 2b and Additional file 3: Figure S2).

Next, we analyzed changes in the structure of mature biofilms formed by $\mathrm{Ab}_{\mathrm{IC}}{ }_{\mathrm{I}} \Delta b l p 1$ and $\mathrm{Ab}_{\mathrm{IC}}{ }_{\mathrm{II}} \Delta b l p 1$ strains and their parental strains after growth for $24 \mathrm{~h}$. The $b l p 1_{\text {IC I }}$ gene deletion resulted in the reduced thickness of the layer of viable cells in a mature biofilm formed by IC I strain and in an increased amount of PI-stained layer, which most likely represents the extracellular DNA (eDNA) producing cells [23] (Fig. 2c, upper panels). Notably, the structure of a mature biofilm formed by $\mathrm{Ab}_{\mathrm{IC}}$ II strain differed from that produced by $\mathrm{Ab}_{\mathrm{IC}}$ I strain, evident through a non-uniform appearance of PI-stained zones, although no apparent differences in the biofilm structure were observed between $\mathrm{Ab}_{\mathrm{IC}}{ }_{\mathrm{II}} \Delta b l p 1$ strain and its parent (Fig. 2c, lower panels). Clearly visible upper PI-stained layer in $\mathrm{Ab}_{\mathrm{IC}}$ II biofilm, which was absent in $A b_{I C}$, indicates the different biofilm maturation compared to $\mathrm{Ab}_{\mathrm{IC}}$ I strain.

We have noticed that $A b_{\text {IC II }}$ strain tended to rapidly form multi-layered clusters in $2 \mathrm{~h}$ after seeding (Fig. 2a). Further maturation of these clusters accumulating a high amount of eDNA, possibly could provide an efficient protection against environmental stress, as this behavior was described for other pathogenic bacteria [24]. Therefore, the results, presented above suggest, that $b l p 1$ allele variants have distinct impact on the biofilm formation by $A$. baumannii IC I and IC II strains.

The blp 1 gene is required for the adhesion of $A b_{1 C}$, strain to lung epithelium cells

To investigate the role of IC-associated blp1 variants on the adhesion phenotype, strains comprising $A b_{I C}$ I and $\mathrm{Ab}_{\mathrm{IC}}$ II , their blp1 deletion mutants and $\Delta b l p 1$ strains complemented with plasmids containing respective blp1 allelic variants were tested for the adhesive properties using mouse lung epithelium LL/2 cells (see Materials and Methods). The blp1 deletion resulted in a reduction 

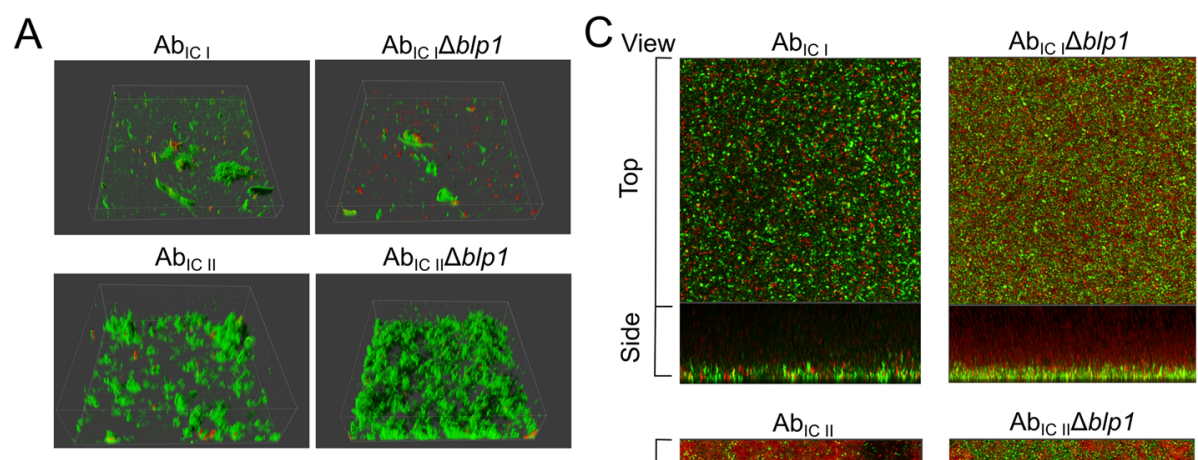

$\mathrm{B}$
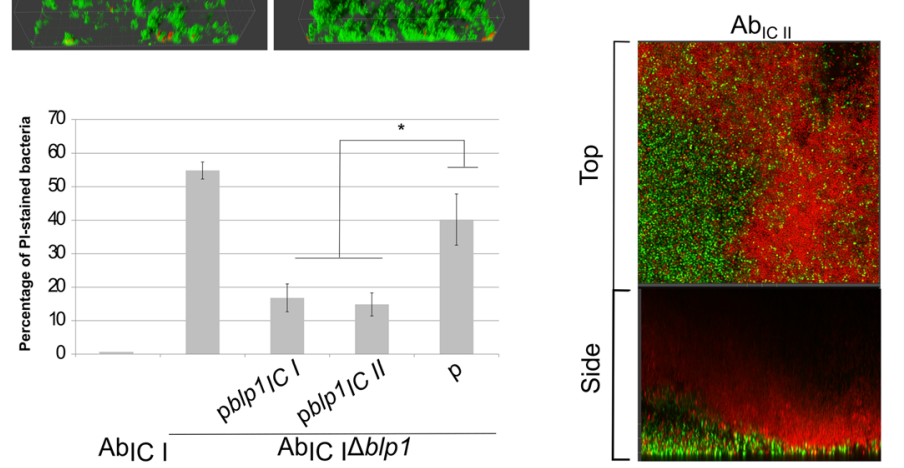

$A b_{I C \|} \Delta b / p 1$

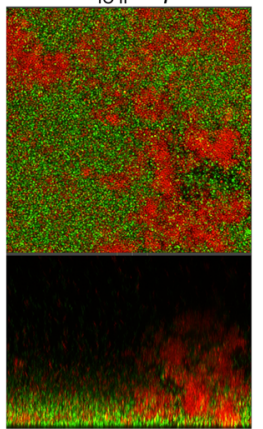

Fig. 2 CLSM analysis of biofilms formed by the A. baumannii IC I and IC II strains. a - 3D visualization of initial attachment to the plastic by A. baumannii blp 1 gene deletion mutants and parental strains assessed after $2 \mathrm{~h}$ of incubation; bacteria were stained with SYTO9 (green) and propidium iodide (red). $\mathbf{b}$ - number of propidium iodide stained bacteria after $2 \mathrm{~h}$ of incubation, compared to the total amount of cells and expressed as a percentage; error bars represent standard errors from the measurements of six different CLSM pictures, significance was assessed by $t$-test, $\left({ }^{*} P<0.05\right)$. c - mature biofilm formation after $24 \mathrm{~h}$ of incubation; $A$. baumannii parental strains and blp 1 gene deletion mutants were stained with SYTO9 (green) and propidium iodide (red); representative top and side views are given

of adhesion of $\mathrm{Ab}_{\mathrm{IC}}{ }_{\mathrm{I}} \Delta b l p 1$ strain by three-fold (Fig. 3, left panel), whereas the loss of blp1 allele in IC II strain had no effect on the adhesion (Fig. 3, right panel). Nevertheless, while dispensable for the adhesion of $\mathrm{Ab}_{\mathrm{IC}}$ II strain, the $b l p 1_{\text {IC II }}$ allele was able to restore adhesive properties of $\mathrm{Ab}_{\mathrm{IC}} \mathrm{I} \Delta b l p 1$ mutant at the level, similar to that of cognate version of blp1 thereby indicating functionality of both $b l p 1$ variants (Fig. 3, left panel).

Therefore, both blp1 gene variants encode proteins that are functional in supporting adhesion of $A$. baumannii to lung epithelium cells, though blp1 deletion in $A b_{\text {IC II }}$ strain had no impact on this phenotype.

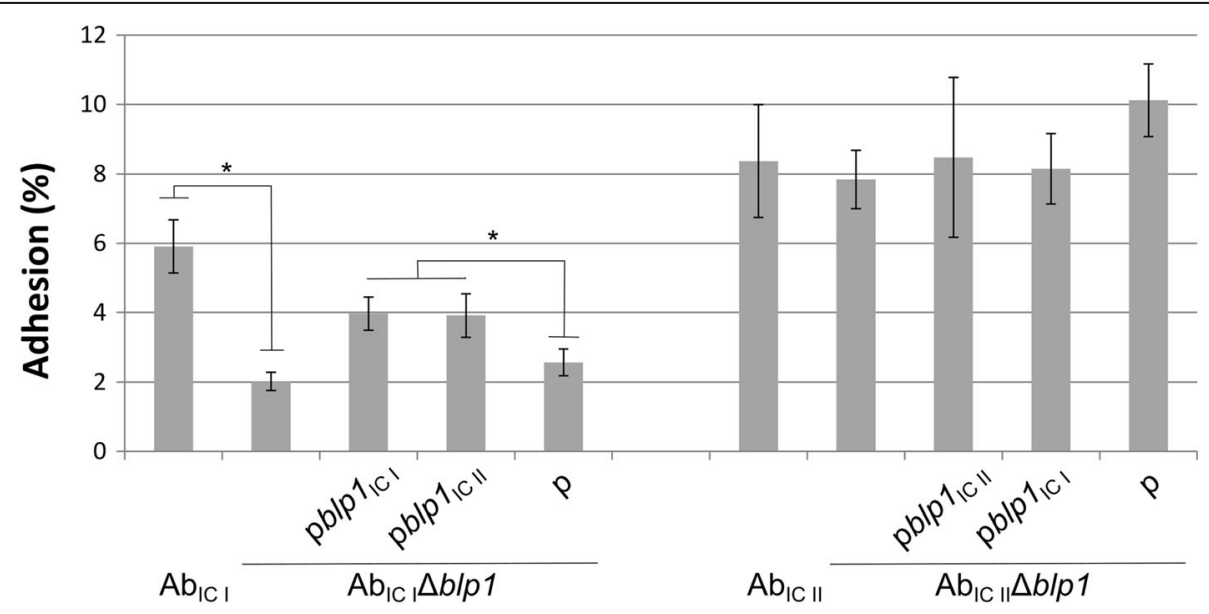

Fig. 3 Adhesion of A. baumannii IC I and IC II strains to the lung epithelium LL/2 cells. Bacterial adhesion to the LL/2 cells after $1.5 \mathrm{~h}$ of incubation was expressed as a percentage of the CFUs of adhered bacteria compared to the total number of CFUs of the initial inoculum; error bars represent standard errors from at least three independent experiments; significance was assessed by $t$-test, $\left({ }^{*} P<0.05\right)$ 
Loss of blp1 gene affects $A$. baumannii virulence in Caenorhabditis elegans and murine infection models

The observed unequal role of blp1 alleles in biofilm formation and cell adhesion prompted us to evaluate their importance in A. baumannii host colonization. For this purpose, C. elegans fertility model was applied as described in Materials and Methods section. As can be seen in Fig. 4a, blp1 deletion in both $\mathrm{Ab}_{\mathrm{IC}}$ and $\mathrm{Ab}_{\mathrm{IC}} \mathrm{II}$ strains resulted in approximately 1.5 -fold increase of worm progeny compared to that observed when C. elegans was infected with the parental strains, indicating reduced virulence in $b l p 1$ deletion mutants.

Both blp1 variants when present on the plasmids were able to restore impaired virulence of $\mathrm{Ab}_{\mathrm{IC}}{ }_{\mathrm{II}} \Delta b l p 1$ strain manifested by the decrease in worm progeny numbers.
Interestingly, the virulent properties of $\mathrm{Ab}_{\mathrm{IC}}{ }_{\mathrm{I}} \Delta b l p 1$ strain demonstrated towards $C$. elegans, were complemented with the cognate $b l p 1$ allele only (Fig. 4a).

Next, we investigated the role of blp1 gene in A. baumannii ability to colonize a vertebrate host. For this purpose, the $A b_{\text {IC I }}$ strain and its $\Delta b l p 1$ mutant were used for the infection. BALB/c mice were infected intraperitoneally and the bacterial burden in the spleens was examined upon $6 \mathrm{~h}$ after infection as described in Materials and Methods. As can be seen in Fig. 4b, approximately three-fold reduction in bacterial loads was observed in mice infected with $A b_{{ }_{I C}}{ }_{I} \Delta b l p 1$ strain compared with those treated with the parental strain indicating the importance of A. baumannii blp1 gene in establishing infection within the host.
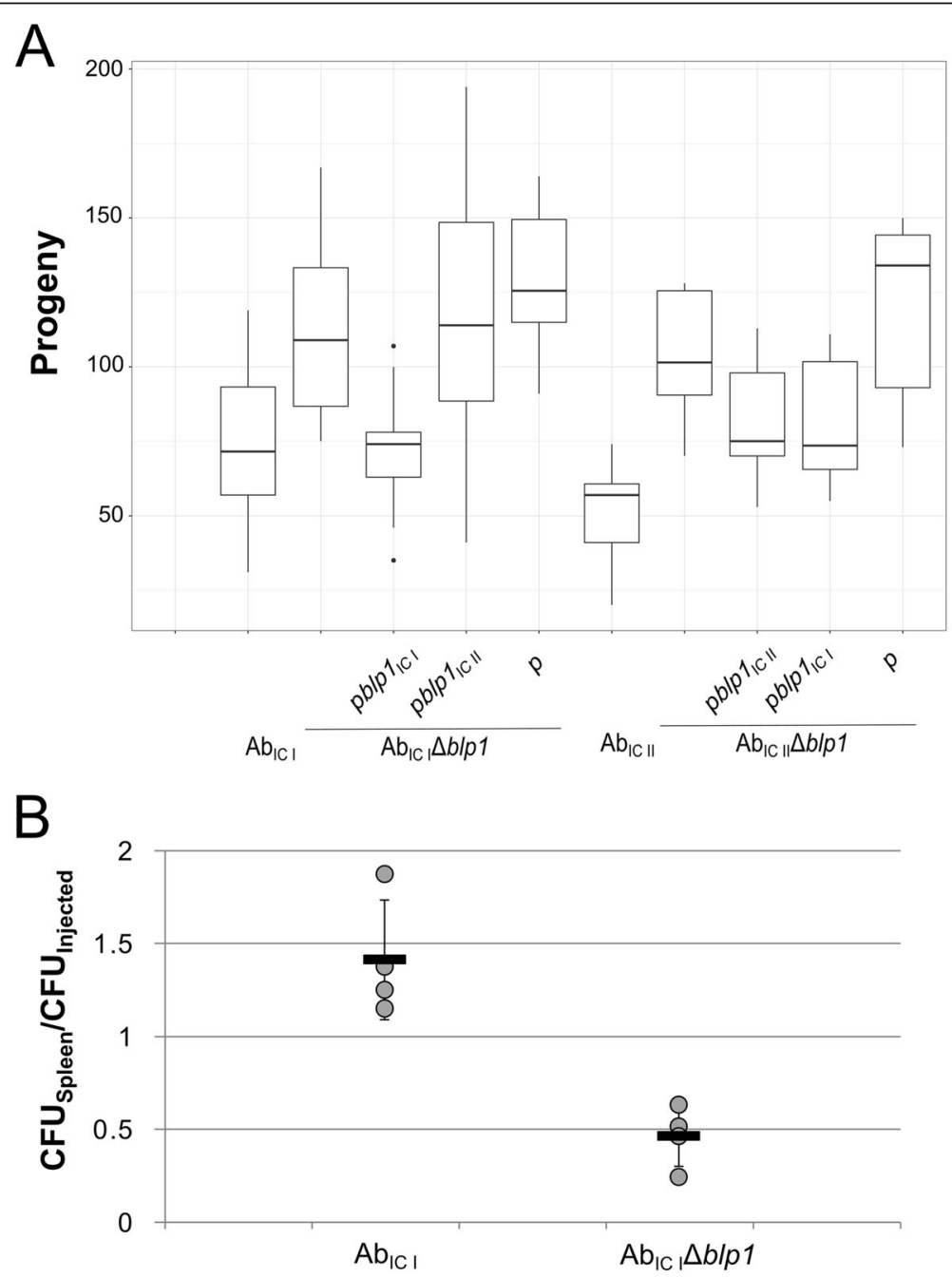

Fig. 4 A. baumannii virulence in animal infection models. a - C. elegans fertility was evaluated after 3 days of nematodes growth in the presence of A. baumannii bacteria on NGM plates; box plot represents the count of nematodes progeny after incubation; data are given from at least two independent experiments, three plates were used in the each experiment; black lines represent medians and whiskers - minimum to maximum values. $\mathbf{b}$ - mice sepsis infection model represented as relative amount of A. baumannii CFUs in the spleens from BALB/c mice $6 \mathrm{~h}$ after infection compared to the injected amount of total CFUs ( $n=4$ mice per group); mice were intraperitoneally infected with $4 \times 10^{5} \mathrm{CFU}$ and $9.5 \times 10^{5} \mathrm{CFU}$ of $A b_{1 C}$, and $A b_{1 C} \Delta b / p 1$, respectively; each dot represents one mouse, black lines indicate averages, whiskers represent standard deviations 
Immunization with C-terminal fragment of Blp1 protein protects from the lethal $A$. baumannii infection in mice Our observation that A. baumannii Blp1 proteins, encoded by blp1 gene variants, share conservative Cterminal 160 amino acid fragment, led us to propose, that it could represent a suitable antigen candidate for investigation of immune-stimulatory properties of Blp1 protein. To increase the number of antigenic moieties displayed at the likely surface-exposed C-terminus of Blp1, 711 amino acids of C-terminal Blp1 from $\mathrm{Ab}_{\mathrm{IC}} \mathrm{I}$ (residues 2652-3362) with a $\mathrm{N}$-terminus His-Tag was purified by affinity chromatography as described in Materials and Methods (Additional file 4: Figure S3).

First, the cytotoxicity of purified recombinant Blp1 fragment has been evaluated using mouse lung epithelium LL/ 2 cells. The purified antigen demonstrated a mild suppressing effect on the growth of $L L / 2$ cells in vitro in the concentration range from 2.5 to $10 \mu \mathrm{g} / \mathrm{ml}$ allowing the maintenance of approximately $90-83 \%$ viability of the cells for 24h (Fig. 5a). Mild cytotoxicity of purified Blp1specific antigen towards lung epithelium cells suggests that it represents a safe candidate antigen for vaccination.

For the active immunization, the $2 \mu \mathrm{g}$ of the recombinant Blp1 fragment were injected into BALB/c mice $(n=$ 5 per group) intramuscularly at the frequency of every 2 weeks, three times in total. At the fourth day after the last immunization, the blood samples were taken and obtained antisera were used for the determination of Blp1-specific-IgG titer by ELISA. The obtained results showed the induction of specific IgG response in the animal group immunized with Blp1 specific antigen compared to the control group (Fig. 5b). Then, on day 42nd, the intraperitoneal challenge with $A$. baumannii $\mathrm{Ab}_{\mathrm{IC}}$ I strain $\left(10^{8} \mathrm{CFU}\right.$ per mouse) has been undertaken and the mice survival rates were monitored for 7 days. The animal group, which received Blp1 specific antigen, demonstrated the $60 \%$ survival rate, whereas no survival in the control group was recorded (Fig. 5c).
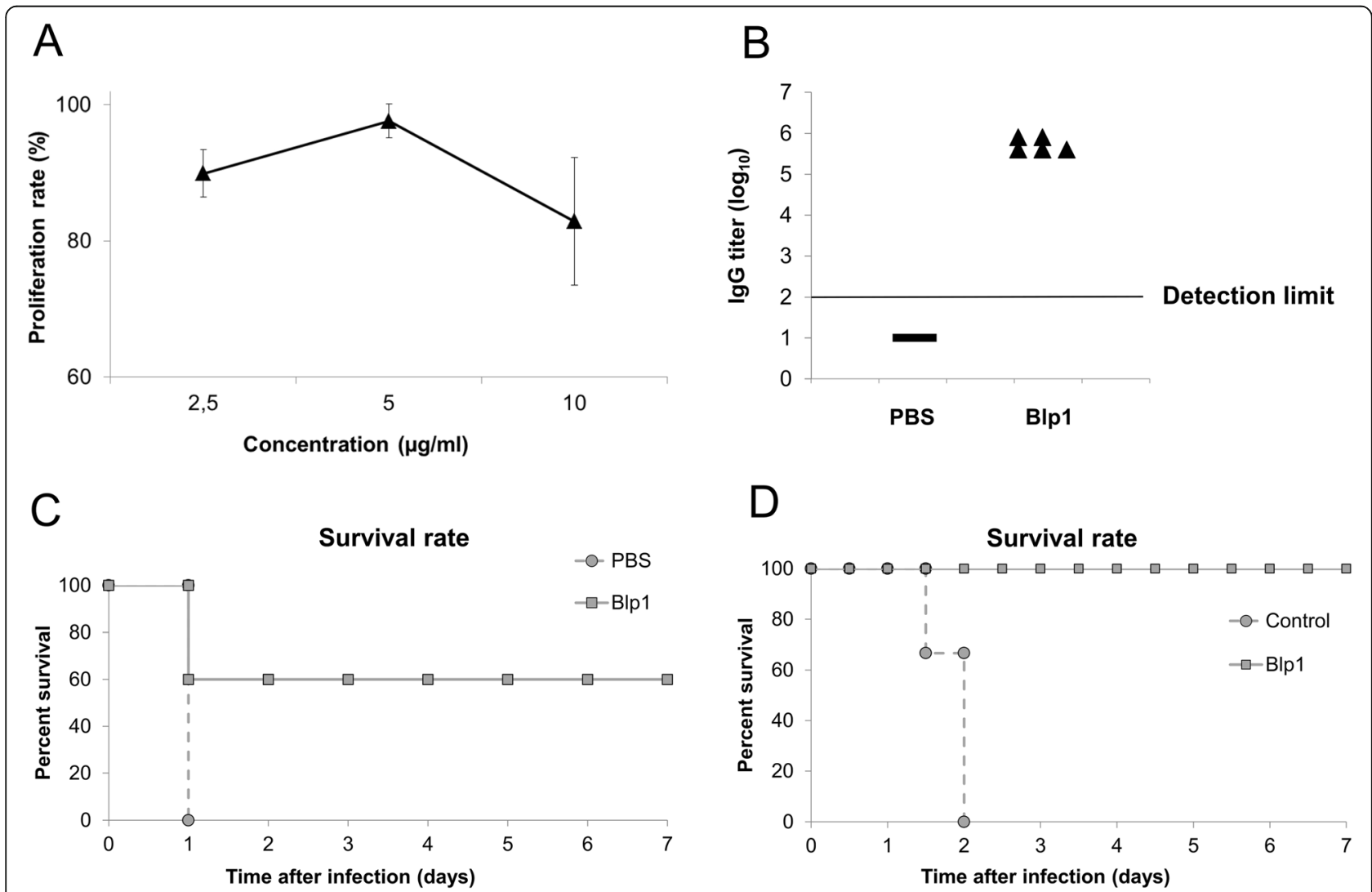

Fig. 5 The effects of Blp1 C-terminal fragment as an immunization agent. a - proliferation rates of LL/2 cells were determined by MTS assay after $24 \mathrm{~h}$ of incubation with different concentrations of recombinant Blp1 fragment; error bars represent standard deviations from three repeats; $\mathbf{b}$ titers of Blp1-specific IgG in mice were detected by ELISA ( $n=5$ mice per group); naive sera obtained from mice, that received adjuvant with PBS were used as a control; horizontal line represents detection limit of the assay. $\mathbf{c}$ - mice survival rates after active vaccination using A. baumannii sepsis model; mice ( $n=5$ mice per group) were challenged intraperitoneally with $10^{8}$ CFUs of Ab $1 \mathrm{C}$, and were monitored for 7 days; $\mathbf{d}$ - passive vaccination effect on the mice survival using $A$. baumannii sepsis model; control group received the serum obtained from mice, immunized with PBS and Freund's adjuvant; mice ( $n=3$ mice per group) were challenged intraperitoneally with $5 \times 10^{7} \mathrm{CFU}$ of $\mathrm{Ab}_{\mathrm{IC}}$ । and were monitored for 45 days 
For the passive immunization, the antisera, obtained from the mice immunized with Blp1 specific antigen and from the control group, treated with PBS, were used. Antisera were injected intraperitoneally into the naïve mice ( $n=3$ per group). Six hours later, animals were challenged with $A$. baumannii by inoculating $5 \times 10^{7}$ CFUs of $A b_{\text {IC I }}$ strain. The survival was monitored for 45 days. As can be seen in the results presented in Fig. 5d, the group, which received antiserum against Blp1 specific antigen, yielded $100 \%$ survival rate, whereas group of mice, that received serum from the control group, resulted in $0 \%$ survival indicating an efficient protective immunity to $A$. baumannii infection, exerted by Blp1 specific antiserum.

\section{Antiserum against Blp1 C-terminal fragment induces opsonophagocytic killing of $A$. baumannii}

To identify the main mechanism responsible for antimicrobial activity of obtained Blp1-antiserum, the A. baumannii $\mathrm{Ab}_{\mathrm{IC}}$ and $\mathrm{Ab}_{\mathrm{IC}}$ II strains were incubated for $1 \mathrm{~h}$ with heat-treated (to inactivate complement components) Blp1-specific and naïve serum in the presence or absence of J744 macrophages (see Materials and Methods). The presence of macrophages increased killing against both $A b_{I C}$ I and $A b_{I C}$ II strains by approximately $20 \%$, when inactivated Blp1-antiserum was present and compared to naïve serum (Fig. 6). In the absence of macrophages, killing efficiency of $A$. baumannii was negligible regardless the origin of inactivated antisera.

Therefore, an increase of opsonophagocytic killing of A. baumannii using heat-inactivated Blp1-antiserum indicates that the antimicrobial activity is macrophagemediated and not complement-depended.

\section{Discussion}

The A. baumannii Blp1 protein belongs to RTX (Repeats-in-toxin) domain-containing protein family [25], however its cellular location is not known. The presence of the T1SS recognition sequence at the very end of Cterminus indicates the secretion of Blp1 via the type I secretion system. The predicted structural organization of $A$. baumannii Blp1 protein such as the presence of multiple Big-like repeats, suggests that it displays properties, which are similar to those observed in extended bacterial fimbrial type adhesins $[9,12,14]$. Indeed, the requirement of $A$. baumannii blp1 gene for the adhesion of IC I strain AYE to the A549 lung epithelium cells was recently reported [11]. We performed a homology search using Swiss-Model platform and the results revealed giant surface-exposed proteins such as SiiE from Salmonella enterica and MpIBP from Marinomonas primoryensis as A. baumannii Blp1 structural homologues (data not shown). Based on the structural investigations, the features of potential adhesin, anchored on the surface of bacterium via its $\mathrm{N}$-terminus have been proposed for Salmonella enterica SiiE protein [12]. Similarly, the $\mathrm{N}$-terminal domains of $M p$ IBP protein have been suggested to anchor the ice and diatom binding adhesin to the outer membrane of M. primoryensis [14]. Our in silico analysis revealed a short (18 residues) $\alpha$-helix as a potential transmembrane sequence at the $\mathrm{N}$-terminus of A. baumannii Blp1 protein (residues 120-137), which

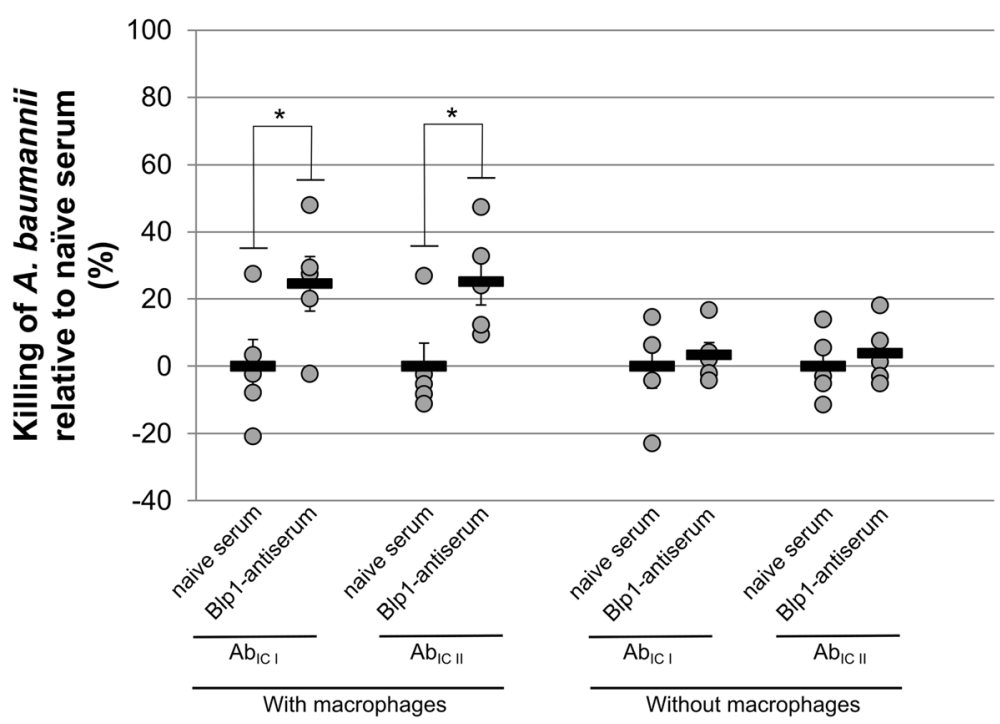

Fig. 6 BIp1-antiserum-depended opsonophagocytic killing of A. baumannii IC I and IC II strains. The bactericidal killing activities of heat-treated Blp1-antiserum and heat-treated naïve serum were analyzed on A. baumannii $A b_{1 C}$, and $A b_{1 C} \|$ strains in the presence and absence of $J 774$ macrophages; each dot represents one mouse, black lines indicate averages and whiskers represent standard errors; significance was assessed by t-test, $\left({ }^{*} P<0.05\right)$ 
could anchor the protein in A. baumannii outer membrane, however, additional investigations are needed to determine the exact mechanism of $A$. baumannii Blp1 immobilization on the surface of bacteria.

Our bioinformatic analysis did not reveal any specific adhesive modules at the $\mathrm{C}$-terminus of $A$. baumannii Blp1, similar to those present at the C-terminus of its structural homolog such as in M. primoryensis MpIBP [14]. However, this study demonstrated the surface adhesion-related properties of Blp1 protein that strongly indicate its role as a bacterial adhesin.

Consistent with the previous study using A. baumannii IC I strain AYE [11], the deletion of a large blp1 gene affected the structure of biofilm formed by $\mathrm{Ab}_{\mathrm{IC}}$ I strain of the common ST231 serotype. The $\mathrm{Ab}_{\mathrm{IC}} \mathrm{I} \Delta b l p 1 \mathrm{mu}-$ tant formed areas with reduced biofilm thickness and increased PI-stained layer. Similar phenotype of impaired biofilm formation was observed for A. baumannii IC I strain AB307-0294, which lacked gene coding for Bap, a giant surface-associated protein [26]. We have observed the PI-stained bacteria of $A b_{I C} \Delta b l p 1$ strain at the initial phase of biofilm formation. The early accumulation of PI-stained bacteria might indicate an abnormal biofilm maturation, as a consequence of the absence of bacterial secreted factors needed for biofilm structure formation. The lack of blp1 could result in liberation of eDNA, as an additional reinforcement material for the extracellular matrix [23]. Interestingly, the impairment of the biofilm formation was observed for $\mathrm{Ab}_{\mathrm{IC}}{ }_{\mathrm{I}} \Delta b l p 1$ mutant only, whereas the deletion of its counterpart in IC II strain had no effect suggesting a different role of blp1 variants on the biofilm-related phenotype in these strains. Moreover, the same trait of different impact of blp1 alleles was clearly seen in the adherence of IC I and IC II strains to lung epithelium cells. However, both blp1 alleles were able to restore phenotypes at a similar level in adhesion-defective $\mathrm{Ab}_{\mathrm{IC}}{ }_{\mathrm{I}} \Delta b l p 1$ mutant. As the blp1 gene transcription was confirmed in both IC I and IC II strains (Additional file 2: Figure S1), the observed differences could be explained by the redundancy of blp 1 for tested adhesion-related phenotype of IC II strain, possibly through the contribution of other extracellular components. The significant differences in biofilm formation were observed comparing A. baumannii IC I strain AB5075 and IC II strain ACICU [27]. The cohesion of type IV pili in A. baumannii ACICU strain was suggested as a reason of bacterial aggregates, similar to

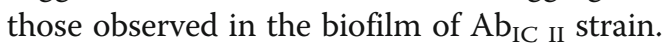

In contrast to biofilm formation and adhesive properties, our study showed the importance of both blp1 variants for the host colonization of respective strains and, in addition, the requirement of the cognate allele for the manifestation of this phenotype in IC I strain. Several studies have demonstrated the allele-dependent variations in virulent properties of bacterial pathogens $[19,28,29]$. Therefore, the Blp1 may possess yet unknown function required for host colonization, different from that mediating the adhesive properties, which appear to be redundant in IC II strain. The genomes of $A$. baumannii IC II strains were found to be more variable, frequently coding for new genes or genes with a higher sequence divergence, which increases a pan-genome ratio, compared to the genomes of IC I strains [15-17]. Given the fact, that IC II strains outspread the IC I strains in the hospital environment [30,31], it is likely that the flexibility of genome, allowing to accumulate new variants and combination of genes, could be the source of the advantage demonstrated by A. baumannii IC II strains, rather than the evolvement of particular protein variants with increased virulent properties.

One of the most promising strategies against MDR $A$. baumannii infections is a development of vaccines [6, 32]. The capsular and surface polysaccharides were researched as potent immunization agents [33, 34], however, their high diversity among $A$. baumannii clinical strains [35] limits development of an universal vaccine. The inactivated whole A. baumannii cells and outer membrane vesicles (OMVs) also demonstrated the induction of immune-response using murine models [36-38]. Compared to the complex antigen mixtures, the pure proteins as vaccine antigens are more desirable due to the safety concerns [39]. Indeed, several $A$. baumannii outer membrane proteins have been used for vaccine research, mostly the abundant outer membrane proteins [40-42]. However, the limitation is that the vast majority of clinical $A$. baumannii strains possess a thick outer layer of polysaccharides (capsule), which efficiently protects pathogens from the host immunity $[7,8]$ by shielding antigens, present on the cell surface of the pathogen. Therefore, the surface-exposed proteins, penetrating the capsule layer, might show superiority as candidates for vaccine development [43].

The high molecular mass of $A$. baumannii Blp1 and adhesion-related phenotype observed for blp1 gene, suggest that Blp1 could penetrate the capsular polysaccharide layer. Blp1 C-terminus possesses a conservative fragment consisting of 160 amino acids, and represents a common antigen displayed by the most prevalent $A$. baumannii strains circulating in the clinical environment. Therefore, the features of Blp1, discussed above, constitute a favorable combination for its role as an immunogen. Differently from the other proposed $A$. baumannii antigens [40-42], this study characterizes a highly prevalent $A$. baumannii antigen, which, due to its high molecular mass, could possibly penetrate the bacterial capsule. This is a substantial advantage considering therapeutic applications, since $A$. baumannii uses capsular polysaccharides as its major virulence factor against host defence system [7, 8]. Importantly, for the 
evaluation of Blp1 immunostimulatory properties, we have used a common A. baumannii strains ST231 (IC I) and ST208 (IC II) and demonstrated, using both active and passive immunizations, that Blp1-specific antigen is a suitable vaccine candidate. The Blp1-antiserum induced an opsonophagocytic killing of IC I and IC II strains, indicating the stimulation of an immune response against clonally disseminated MDR A. baumannii. It is worth to mention, that since the immunisation trials were performed with limited number of mice, there is a need for further investigations using larger amount of samples in order to extend this study.

\section{Conclusions}

In this study we have investigated the A. baumannii surface-exposed protein Blp1 with an emphasis on its role in virulence, expressed by clinical $A$. baumannii strains and its suitability as a drug target. We have observed differences between IC strains, which should be taken into consideration, when the new drugs are investigated. Ideally, for the development of vaccine candidates, the most prevalent $A$. baumannii strains, currently circulating in the clinical environment should be used, since this opportunistic pathogen is well known for its genome variability.

\section{Methods}

Bacterial strains and growth conditions

Strains used in this study are listed in the Additional file 1: Table $\mathrm{S} 1 . \mathrm{Ab}_{\mathrm{IC} \text { I }}$ and $\mathrm{Ab}_{\mathrm{IC} \text { II }}$ strains are representative strains corresponding to IC I and IC II, respectively. Strains were isolated from Lithuanian University of Health Sciences Kauno Klinikos Hospital in 2010 and assigned to IC I and IC II by trilocus sequence-based typing (3LST) as described previously [20]. Clinical $A$. baumannii strains were cultured on Luria-Bertani (LB) agar plates at $37^{\circ} \mathrm{C}$. Liquid cultures were inoculated in LB media overnight.

\section{PCR}

One hundred twenty-two clinical $A$. baumannii strains belonging to either IC I $(n=72)$ or IC II $(n=50)$ clonal lineage were screened for the presence of blp1 alleles. Conventional PCR was undertaken using primers listed in Additional file 1: Table S1. Tm was calculated based on the primer sequences.

For $b l p 1$ gene transcription analysis, clinical $A$. baumannii strains were grown in LB medium for $16 \mathrm{~h}$. Total RNA was extracted using GeneJET RNA Purification Kit (Thermo Fisher Scientific) according to the manufacturer recommendations. cDNA was synthesized using RevertAid First Strand cDNA Synthesis Kit (Thermo Fisher Scientific). qPCR was performed using primer pairs listed in Additional file 1: Table S1.

\section{Generation of $A$. baumannii $\Delta b / p 1$ mutants and complemented strains}

Markerless gene deletion was performed as previously described [44]. Briefly, upstream and downstream regions of A. baumannii blp1 gene were amplified using primers listed in Additional file 1: Table S1. The amplicons were joined with gentamicin resistance cassette aac3I by overlap PCR. The resulting DNA was cloned into pUC19_sacB plasmid yielding pUC19_sacB UDblp1Gm plasmid (Additional file 1: Table S1). A. baumannii strains were transformed with the resulting plasmid by electroporation and colonies were selected on LB agar with gentamicin. Colonies were inoculated in LB medium for $4 \mathrm{~h}$. Serial dilutions were plated on LB agar with $10 \%$ sucrose. For the complementation, the DNA comprising the blp1 gene with an upstream region including a putative promoter region was amplified using primers listed in Additional file 1: Table S1 and DNA from $A b_{I C}$ and $A b_{I C}$ II strains as a template. The resulting DNA fragments were cloned into pUC19_gm AcORI plasmid and selected in E. coli JM107 strain (Additional file 1: Table S1). The $\mathrm{Ab}_{\mathrm{IC}}{ }_{\mathrm{I}} \Delta b l p l$ and $\mathrm{Ab}_{\mathrm{I}-}$ ${ }_{\mathrm{CII}} \Delta b l p 1$ strains were transformed with the resulting plasmids $\mathrm{p} b l p 1_{\mathrm{IC} \text { I }}$ and $\mathrm{p} b l p 1_{\mathrm{IC} \text { II }}$, respectively, by electroporation and colonies were selected on LB agar with $30 \mu \mathrm{g} / \mathrm{ml}$ gentamicin. Generated mutants and recombinant constructs were confirmed by sequencing.

\section{Confocal laser scanning microscopy (CLSM)}

For evaluation of biofilms, 1000-fold dilutions of overnight $A$. baumannii cultures were used for seeding into LB media. Biofilms were grown for 2 and $24 \mathrm{~h}$ at $37^{\circ} \mathrm{C}$ without agitation. After growth in micro-titer plates, biofilms were stained for $2 \mathrm{~h}$ by the Filmtracer TM LIVE/ DEAD $^{\circ}$ Biofilm Viability Kit (Thermo Fisher Scientific). The plate was then placed on the motorized stage of an inverted confocal microscope (TCS SP8 AOBS, Leica Microsystems) at the INRA-MIMA2 imaging platform as described by [45].

\section{Cell culture assays}

Mouse epithelial LL/2 (LLC1) and mouse macrophages J774 cell lines were grown in Dulbecco's modified Eagle's medium (DMEM) (Gibco) supplemented with 10\% fetal bovine serum (FBS) (Gibco) at $37^{\circ} \mathrm{C}$ with $5 \% \mathrm{CO}_{2}$. Adhesion experiments were performed as described by [8]. Bacterial adherence to the LL/ 2 cells was expressed as a percentage of the CFU of adhered bacteria compared to the total number of CFUs of the initial inoculum.

For the opsonophagocytic killing assay, mouse macrophages $\mathrm{J774}$ were stimulated with $40 \mathrm{ng} / \mathrm{ml}$ E. coli LPS (Sigma-Aldrich) for 3 days. Macrophages $\left(1 \times 10^{5}\right.$ cells/ well $)$ and $A$. baumannii strains $\left(1 \times 10^{4} \mathrm{CFU} /\right.$ well $)$ were added into the wells along with the heat-treated mice 
serum $\left(56^{\circ} \mathrm{C}\right.$ for $40 \mathrm{~min}$ for the inactivation of complement components) at the final dilution of 1:200. After 1 $\mathrm{h}$ incubation, the samples were serially diluted and seeded. Serum killing rates were counted by comparing the number of the reduced CFUs with those observed using naïve serum.

A cytotoxicity assay was performed by incubating LL/2 cells with recombinant Blp1 C-terminal fragment in DMEM supplemented with $10 \%$ FBS at $37^{\circ} \mathrm{C}$ for $24 \mathrm{~h}$. Then, $20 \mu \mathrm{l}$ of MTS (Promega) solution was added to the wells and cells were incubated for $4 \mathrm{~h}$ at $37^{\circ} \mathrm{C}$. After incubation, $\mathrm{OD}_{490}$ was determined. The proliferation rate was counted by comparing $\mathrm{OD}_{490}$ values for $\mathrm{Blp} 1$ $\mathrm{C}$-terminus fragment-treated and non-treated cells.

\section{C. elegans fertility assay}

A. baumannii strains were investigated using C. elegans fertility model as described previously [8]. Overnight cultures of different A. baumannii strains were seeded on NGM medium. One L2 stage worm was placed over each $A$. baumannii strain and incubated at $21.5^{\circ} \mathrm{C}$. On the third day after infection worm progeny was determined by counting C. elegans worms.

\section{Cloning and protein purification}

The DNA of Blp1 C-terminal fragment spanning 26523362 amino acids of blp1 coding region was amplified using primer pair BldBamF/BlXhR (Additional file 1: Table S1). The resulting amplicon was cloned into pET-28b expression plasmid by fusing $6 \mathrm{xHis}$ tag sequence to the $\mathrm{N}$ terminus of the recombinant protein. The resulting plasmid was sequenced and transformed into the expression host strain E. coli ArcticExpress (DE3) (Additional file 1: Table S1). Culture was grown in LB broth containing $40 \mu \mathrm{g} / \mathrm{ml}$ of kanamycin to $\mathrm{OD}_{600}$ of 0.5 . Protein expression was induced by adding $0.5 \mathrm{mM}$ of IPTG and incubating at $14^{\circ} \mathrm{C}$ for 16 h. Cells were suspended in lysis buffer $\left(20 \mathrm{mM} \mathrm{NaH}_{2} \mathrm{PO}_{4}\right.$ $\mathrm{pH} 7.4,500 \mathrm{mM} \mathrm{NaCl}, 20 \mathrm{mM}$ imidazole) supplemented with protease inhibitor PMSF, disrupted by sonication and centrifuged $12,000 \mathrm{x} \mathrm{g}$ at $4{ }^{\circ} \mathrm{C}$, for $30 \mathrm{~min}$ to remove insoluble material. Proteins were purified from soluble fraction by affinity chromatography, using $1 \mathrm{ml}$ HisTrapHPTM nickel-Sepharose column (GE Healthcare). Proteins were eluted by a linear gradient using buffer $20 \mathrm{mM} \mathrm{NaH}_{2} \mathrm{PO}_{4}$ $\mathrm{pH}$ 7.4, $500 \mathrm{mM} \mathrm{NaCl}, 500 \mathrm{mM}$ imidazole. The eluted fractions were desalted using Sephadex G-25 (GE Healthcare) column, exchanging to PBS buffer.

\section{ELISA}

Antigen-specific ELISA was performed to quantify the anti-Blp1 antibody response in sera from vaccinated mice as described before [46]. 96-well Maxisorp plates (Nalge Nunc) were coated with $0.2 \mu \mathrm{g}$ Blp1 fragment/ well by incubation at $4{ }^{\circ} \mathrm{C}$ in PBS overnight. The titer of specific IgG was established using horseradish peroxidase substrate (Thermo Fisher Scientific) and determining the absorbance at $450 \mathrm{~nm}$.

\section{Murine models}

Eight to 12 weeks old female BALB/c mice were purchased from Institute of Biochemistry, Life Science Center (Vilnius University, Vilnius). The animals were maintained and used in accordance with the recommendations of the directive 2010/63/EU of the European Parliament and of the Council of 22 September 2010 on the protection of animals used for scientific purpose. Study was performed under permission of Lithuanian State Food and Veterinary Service no. G2-72.

A sepsis model was established as described previously [37]. Briefly, A. baumannii cultures were prepared by mixing the bacterial suspension with $5 \%$ of porcine mucin $(\mathrm{w} /$ v; Sigma-Aldrich). BALB/c mice ( $n=4$ per group) were injected intraperitoneally with $0.5 \mathrm{ml}$ of the sample. The CFUs of the corresponding the bacterial loads were determined by plating sequential dilutions on LB plates.

For the immunization experiments, groups of $\mathrm{BALB} / \mathrm{c}$ mice $(n=5)$ were immunized intramuscularly with $2 \mu \mathrm{g}$ of recombinant Blp1 protein fragment. Immunization mixture was prepared by mixing the antigen with an equal volume of complete Freund's adjuvant on the day 0 and with incomplete Freund's adjuvant on the days 14 and 28. Control group was inoculated with PBS combined with Freund's adjuvant. On the day 32, blood samples were collected and tested against immunogen using ELISA as described above. Mice were challenged intraperitoneally on the day 42 with $1 \times 10^{8} \mathrm{CFU}$ of $\mathrm{Ab}_{\mathrm{IC}} \mathrm{I}$ bacteria.

For the passive immunization, the $200 \mu \mathrm{L}$ of antiserum was injected intraperitoneally into naïve mice ( $n=3$ per group). Control group received the serum obtained from mice, immunized with $\mathrm{PBS}$ and Freund's adjuvant. After $6 \mathrm{~h}, 5 \times 10^{7} \mathrm{CFU}$ of $\mathrm{Ab}_{\mathrm{IC}}$ I bacteria were injected intraperitoneally.

After experiments mice were sacrificed using cervical dislocation.

\section{Bioinformatic and statistical analysis}

Bioinformatic analysis was undertaken using genomes obtained from NCBI database. Sequences alignments were made using TexShade tool. Statistical comparisons were performed using equal variance $t$-test.

\section{Supplementary information}

Supplementary information accompanies this paper at https://doi.org/10. 1186/s12866-019-1615-3.

Additional file 1: Table S1. Oligonucleotides, plasmids and strains used in this work. 
Additional file 2: Figure $\mathbf{S} 1$. b/p 1 gene expression in A. baumannii IC and IC II strains.

Additional file 3: Figure S2. CLSM analysis of biofilms formed by the $A$ baumannii strains after 2 and $24 \mathrm{~h}$ of incubation.

Additional file 4: Figure S3. Expression and purification of the recombinant His-Blp1 2652-3362 C-terminal fragment.

\section{Abbreviations}

cDNA: complementary DNA; CFU: Colony-forming unit; CLSM: Confocal laser scanning microscopy; DMEM: Dulbecco's modified Eagle's medium; DNA: Deoxyribose nucleic acid; eDNA: extracellular DNA; ELISA: EnzymeLinked ImmunoSorbent Assay; FBS: Fetal bovine serum; IC: International clone; IPTG: Isopropyl $\beta$-D-1-thiogalactopyranoside; LB: Luria-Bertani; LPS: Lipopolisacharide; MDR: Multidrug resistance; MLST: Multilocus sequence type; NGM: Nematode growth medium; OMV: Outer membrane vesicles; PBS: Phosphate-Buffered Saline; PCR: Polymerase chain reaction; PI: Propidium iodide; qPCR: quantitative PCR; RNA: Ribonucleic acid RTX: Repeats-in-toxin; SDS-PAGE: Sodium dodecylsulfate polyacrylamide gel electrophoresis

\section{Acknowledgements}

Not applicable.

\section{Authors' contributions}

JS designed the experiments. JS, EK, JD, RK, JA, RB performed the experiments. JS and ES analyzed the data and wrote the manuscript. All of the authors critically reviewed the manuscript and approved the final version.

\section{Funding}

Not applicable.

\section{Availability of data and materials}

The datasets used and analyzed during the current study are available from the corresponding author on reasonable request.

\section{Ethics approval and consent to participate}

The animals were maintained and used in accordance with the recommendations of the directive 2010/63/EU of the European Parliament and of the Council of 22 September 2010 on the protection of animals used for scientific purpose. Study was performed under permission of Lithuanian State Food and Veterinary Service no. G2-72

\section{Consent for publication}

Not applicable.

\section{Competing interests}

The authors declare that they have no competing interests

\section{Author details}

${ }^{1}$ Institute of Biosciences, Life Sciences Center, Vilnius University, Sauletekio ave. 7, LT-10257 Vilnius, Lithuania. ${ }^{2}$ Micalis Institute, INRA, AgroParisTech, Université Paris-Saclay, 78350 Jouy-en-Josas, France.

\section{Received: 7 June 2019 Accepted: 17 October 2019}

\section{Published online: 21 November 2019}

\section{References}

1. Harding CM, Hennon SW, Feldman MF. Uncovering the mechanisms of Acinetobacter baumannii virulence. Nat Rev Microbiol. 2018;16(2):91-102

2. Zarrilli R, Pournaras S, Giannouli M, Tsakris A. Global evolution of multidrugresistant Acinetobacter baumannii clonal lineages. Int J Antimicrob Agents. 2013:41(1):11-9.

3. Dahdouh E, Gómez-Gil R, Pacho S, Mingorance J, Daoud Z, Suárez M. Clonality, virulence determinants, and profiles of resistance of clinical Acinetobacter baumannii isolates obtained from a Spanish hospital. PLoS One. 2017;12(4):e0176824.

4. Rodriguez-Martinez JM, Nordmann P, Fortineau N, Poirel L. VIM-19, a metallo-beta-lactamase with increased carbapenemase activity from
Escherichia coli and Klebsiella pneumoniae. Antimicrob Agents Chemother. 2010:54(1):471-6.

5. Isler B, Doi Y, Bonomo RA, Paterson DL. New Treatment Options against Carbapenem-Resistant Acinetobacter baumannii Infections. Antimicrob Agents Chemother. 2018;63(1). https://doi.org/10.1128/AAC.01110-18.

6. Dickey SW, Cheung GYC, Otto M. Different drugs for bad bugs: antivirulence strategies in the age of antibiotic resistance. Nat Rev Drug Discov. 2017; 16(7):457-71.

7. Russo TA, Luke NR, Beanan JM, Olson R, Sauberan SL, MacDonald U, et al. The K1 capsular polysaccharide of Acinetobacter baumannii strain 307-0294 is a major virulence factor. Infect Immun. 2010;78(9):3993-4000.

8. Skerniškytė J, Krasauskas R, Péchoux C, Kulakauskas S, Armalyte J, Sužiedèlienè E. Surface-Related Features and Virulence Among Acinetobacter baumannii Clinical Isolates Belonging to International Clones I and II. Front Microbiol. 2019:9:3116.

9. Lin YP, McDonough SP, Sharma Y, Chang YF. The terminal immunoglobulin like repeats of LigA and LigB of Leptospira enhance their binding to gelatin binding domain of fibronectin and host cells. PLoS One. 2010;5(6):e11301.

10. Epler Barbercheck CR, Bullitt E, Andersson M. Bacterial adhesion Pili. Subcell Biochem. 2018;87:1-18.

11. Gregorio E, Del Franco M, Martinucci M, Roscetto E, Zarrilli R, Di Nocera PP. Biofilmassociated proteins: news from Acinetobacter. BMC Genomics. 2015;16:933.

12. Griessl MH, Schmid B, Kassler K, Braunsmann C, Ritter R, Barlag B, et al. Structural insight into the giant $\mathrm{Ca}^{2+}$-binding adhesion SiiE: implications for the adhesion of Salmonella enterica to polarized epithelial cells. Structure. 2013:21(5):741-52

13. Bodeloón G, Palomino C, Fernández LÁ. Immunoglobulin domains in Echerichia coli and other enterobacteria: from pathogenesis to applications in antibody technologies. FEMS Microbiol Rev. 2013;37:204-50.

14. Guo S, Stevens CA, Vance T, Olijve L, Graham LA, Campbell RL, et al. Structure of a 1.5-MDa adhesin that binds its Antarctic bacterium to diatoms and ice. Sci Adv. 2017:3(8):e1701440.

15. Imperi F, Antunes LC, Blom J, Villa L, lacono M, Visca $P$, et al. The genomics of Acinetobacter baumannii: insights into genome plasticity, antimicrobial resistance and pathogenicity. IUBMB Life. 2011;63(12):1068-74.

16. Snitkin ES, Zelazny AM, Montero Cl, Stock F, Mijares L, NISC Comparative Sequence Program, Murray PR, Segre JA. Genome-wide recombination drives diversification of epidemic strains of Acinetobacter baumannii. Proc Natl Acad Sci U S A. 2011;108(33):13758-63.

17. Eijkelkamp BA, Stroeher UH, Hassan KA, Paulsen IT, Brown MH. Comparative analysis of surface-exposed virulence factors of Acinetobacter baumannii. BMC Genomics. 2014;15:1020.

18. Smith SG, Mahon V, Lambert MA, Fagan RP. A molecular Swiss army knife: OmpA structure, function and expression. FEMS Microbiol Lett. 2007;273(1):1-11.

19. Wu Z, Periaswamy B, Sahin O, Yaeger M, Plummer P, Zhai W, et al. Point mutations in the major outer membrane protein drive hypervirulence of a rapidly expanding clone of Campylobacter jejuni. Proc Natl AcadSci U S A. 2016;113(38):10690-5

20. Povilonis J, Seputiene V, Krasauskas R, Juskaite R, Miskinyte M, Suziedelis K, et al. Spread of carbapenem-resistant Acinetobacter baumannii carrying a plasmid with two genes encoding OXA-72 carbapenemase in Lithuanian hospitals. J Antimicrob Chemother. 2013;68(5):1000-6.

21. Ghaith DM, Zafer MM, Al-Agamy MH, Alyamani EJ, Booq RY, Almoazzamy O. The emergence of a novel sequence type of MDR Acinetobacter baumannii from the intensive care unit of an Egyptian tertiary care hospital. Ann Clin Microbiol Antimicrob. 2017;16(1):34.

22. Chen Q, Zhou JW, Fan JZ, Wu SH, Xu LH, Jiang Y, et al. Simultaneous emergence and rapid spread of three OXA-23 producing Acinetobacter baumannii ST208 strains in intensive care units confirmed by whole genome sequencing. Infect Genet Evol. 2018;58:243-50.

23. Vorkapic D, Pressler K, Schild S. Multifaceted roles of extracellular DNA in bacterial physiology. Curr Genet. 2015;62(1):71-9.

24. Zapotoczna M, O'Neill E, O'Gara JP. Untangling the diverse and redundant mechanisms of Staphylococcus aureus biofilm formation. PLoS Pathog. 2016; 12(7):e1005671.

25. Linhartová I, Bumba L, Mašín J, Basler M, Osička R, Kamanová J, et al. RTX proteins: a highly diverse family secreted by a common mechanism. FEMS Microbiol Rev. 2010:34(6):1076-112.

26. Loehfelm TW, Luke NR, Campagnari AA. Identification and characterization of an Acinetobacter baumannii biofilm-associated protein. J Bacteriol. 2008 190(3):1036-44 
27. Ronish LA, Lillehoj E, Fields JK, Sundberg EJ, Piepenbrink KH. The structure of PilA from Acinetobacter baumannii AB5075 suggests a mechanism for functional specialization in Acinetobacter type IV pili. J Biol Chem. 2019; 294(1):218-30.

28. Mittal R, Krishnan S, Gonzalez-Gomez I, Prasadarao NV. Deciphering the roles of outer membrane protein a extracellular loops in the pathogenesis of Escherichia coli K1 meningitis. J Biol Chem. 2010;286(3):2183-93.

29. Skerniškytė J, Karazijaitė E, Deschamps J, Krasauskas R, Briandet R, Sužiedèlienè $E$. The Mutation of Conservative Asp268 Residue in the Peptidoglycan-Associated Domain of the OmpA Protein Affects Multiple Acinetobacter baumannii Virulence Characteristics. Molecules. 2019;24(10) https://doi.org/10.3390/molecules24101972.

30. Kim DH, Jung SI, Kwon KT, Ko KS. Occurrence of Diverse AbGRI1-Type Genomic Islands in Acinetobacter baumannii Global Clone 2 Isolates from South Korea. Antimicrob Agents Chemother. 2017;61(2). https://doi.org/10. 1128/AAC.01972-16.

31. Pournaras S, Dafopoulou K, Del Franco M, Zarkotou O, Dimitroulia E,

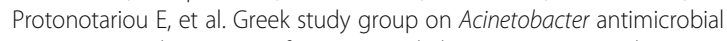
resistance. Predominance of international clone 2 OXA-23-producingAcinetobacter baumannii clinical isolates in Greece, 2015: results of a nationwide study. Int J Antimicrob Agents. 2017;49(6):749-53.

32. Ahmad TA, Tawfik DM, Sheweita SA, Haroun M, El-Sayed LH. Development of immunization trials against Acinetobacter baumannii. Trials in Vaccinology. 2016;5:53-60

33. Bentancor LV, O'Malley JM, Bozkurt-Guzel C, Pier GB, Maira-Litrán T. Poly-Nacetyl- $\beta-(1-6)$-glucosamine is a target for protective immunity against Acinetobacter baumannii infections. Infect Immun. 2012;80(2):651-6.

34. Russo TA, Beanan JM, Olson R, MacDonald U, Cox AD, St Michael F, et al. The $\mathrm{K} 1$ capsular polysaccharide from Acinetobacter baumannii is a potential therapeutic target via passive immunization. Infect Immun. 2013;81(3):915-22.

35. Hu D, Liu B, Dijkshoorn L, Wang L, Reeves PR. Diversity in the major polysaccharide antigen of Acinetobacter baumannii assessed by DNA sequencing, and development of a molecular serotyping scheme. PLoS One. 2013;8(7):e70329.

36. McConnell MJ, Pachón J. Active and passive immunization against Acinetobacter baumannii using an inactivated whole cell vaccine. Vaccine. 2010;29(1):1-5.

37. Huang W, Yao Y, Long Q, Yang X, Sun W, Liu C, et al. Immunization against multidrug-resistant Acinetobacter baumannii effectively protects mice in both pneumonia and sepsis models. PLoS One. 2014;9(6):e100727.

38. KuoLee R, Harris G, Yan H, Xu HH, Conlan WJ, Patel GB, et al. Intranasal immunization protects against Acinetobacter baumannii-associated pneumonia in mice. Vaccine. 2015;33(1):260-7

39. Colombo C, Pitirollo O, Lay L. Recent Advances in the Synthesis of Glycoconjugates for Vaccine Development. Molecules. 2018;23(7). https:// doi.org/10.3390/molecules23071712.

40. Huang W, Yao Y, Wang S, Xia Y, Yang X, Long Q, et al. Immunization with a 22-kDa outer membrane protein elicits protective immunity to multidrugresistant Acinetobacter baumannii. Sci Rep. 2016;6:20724.

41. Singh R, Garg N, Shukla G, Capalash N, Sharma P. Immunoprotective efficacy of Acinetobacter baumannii outer membrane protein, FilF, Predicted In silico as a Potential Vaccine Candidate. Front Microbiol. 2016;7:158.

42. Zhang X, Yang T, Cao J, Sun J, Dai W, Zhang L. Mucosal immunization with purified OmpA elicited protective immunity against infections caused by multidrug-resistant Acinetobacter baumannii. Microb Pathog. 2016;96:20-5.

43. Badmasti F, Ajdary S, Bouzari S, Fooladi AA, Shahcheraghi F, Siadat SD. Immunological evaluation of OMV (PagL)+bap(1-487aa) and AbOmpA(8346aa)+bap(1-487aa) as vaccine candidates against Acinetobacter baumannii sepsis infection. Mol Immunol. 2015;67:552-8.

44. Oh MH, Lee JC, Kim J, Choi CH, Han K. Simple method for Markerless gene deletion in multidrug-resistant Acinetobacter baumannii. Appl Environ Microbiol. 2015;81:3357-68.

45. Poquet I, Saujet L, Canette A, Monot M, Mihajlovic J, Ghigo JM, et al. Clostridium difficile biofilm: remodeling metabolism and cell surface to build a sparse and heterogeneously aggregated architecture. Front Microbiol. 2018;9:2084

46. McConnell MJ, Hanna PC, Imperiale MJ. Cytokine response and survival of mice immunized with an adenovirus expressing Bacillus anthracis protective antigen domain 4. Infect Immun. 2006;74(2):1009-15.

\section{Publisher's Note}

Springer Nature remains neutral with regard to jurisdictional claims in published maps and institutional affiliations.
Ready to submit your research? Choose BMC and benefit from:

- fast, convenient online submission

- thorough peer review by experienced researchers in your field

- rapid publication on acceptance

- support for research data, including large and complex data types

- gold Open Access which fosters wider collaboration and increased citations

- maximum visibility for your research: over $100 \mathrm{M}$ website views per year

At $\mathrm{BMC}$, research is always in progress.

Learn more biomedcentral.com/submissions 